\title{
Coulomb interaction and persistent currents in ensembles of mesoscopic metal rings
}

\author{
G. Vignale \\ Department of Physics, Missouri University, Columbia, Missouri 65211
}

(Received 25 May 1994)

\begin{abstract}
The average persistent current in an ensemble of mesoscopic metal rings at fixed chemical potential threaded by a magnetic flux in the diffusive regime is related to the presence of a component of the oneelectron effective self-energy, which is odd with respect to the electron velocity. In a noninteracting model, the "velocity-odd" component of the self-energy, calculated within the self-consistent Born approximation, is found to be exponentially small. Hence, the average persistent current is also exponentially small. When the Coulomb interaction between electrons is included, a finite velocity-odd component of the self-energy is obtained. The microscopic reason for this is the enhancement of the average classical electrostatic energy of interaction between two electrons with almost opposite velocities. We show that the recent theory of Ambegaokar and Eckern can be reformulated in terms of a velocity-odd self-energy, and their results are reproduced. A simple model is then presented, which exhibits the main qualitative features of their result in an elementary way. Finally, the assumption that all rings have the same chemical potential is examined. It is shown that the error involved in this approximation is parametrically small for three-dimensional rings, scaling as the inverse of the volume of the ring.
\end{abstract}

\section{INTRODUCTION}

Several recent experiments ${ }^{1-3}$ have confirmed an old theoretical prediction ${ }^{4}$ that mesoscopic, nonsuperconducting metal rings, threaded by a magnetic flux $\Phi$, carry a persistent current, which is a periodic function of $\Phi$ with a period of one flux quantum $\Phi_{0}=h c / e$. However, these experiments have also raised a number of new questions that the theory ${ }^{5-11}$ must answer.

First of all, there is the question of the role played by impurities. In the experiment of Lévy et al. ${ }^{1}$ the persistent current was averaged over an ensemble of approximately $10^{7}$ three-dimensional copper rings which were strongly diffusive, i.e., the mean free path $l$ between elastic electron-impurity collisions was much shorter than the length of the ring $L$. The average persistent current was observed to be periodic in $\Phi$ with a period of half a flux quantum, and its magnitude was measured to be of the order of the "diffusion current" $I_{D}=e v_{F} l / L^{2}\left(v_{F}\right.$ is the Fermi velocity) - the current carried by a single electron at the Fermi surface diffusing around the ring. The major theoretical challenge here has been to obtain the correct order of magnitude of the current after averaging over different impurity distributions. We shall see that the Coulomb interaction ${ }^{9-11}$ between electrons is essential to obtaining a result that scales as $I_{D}$. The periodhalving phenomenon, on the other hand, can be explained with a noninteracting ${ }^{7,8}$ as well as an interacting theory.

In the experiment of Chadrasekhar et al. ${ }^{2}$ a single three-dimensional diffusive gold loop was studied. The current was found to be periodic with period $\Phi_{0}$, and to have a value of the order of the "ballistic current" $I_{B}=e v_{F} / L$ - the current carried by a single free electron at the Fermi surface traveling around the ring-about 100 times larger than $I_{D}$. This is a very surprising result because, according to thermodynamics, the current car- ried in the ground state is given by $I=-c \partial E / \partial \Phi$, where $E$ is the ground-state energy. A change in flux is equivalent to a change in boundary conditions on the wave function, and it is well known that the variation of an energy level corresponding to a change from periodic to antiperiodic boundary conditions (which amounts to changing $\Phi$ by $\Phi_{0} / 2$ ) is of the order of the "Thouless energy" $E_{c}=\hbar D / L^{2}$, where $D$ is the diffusion constant. Then we should expect the typical current to be of the order of $c E_{c} / \Phi_{0}=I_{D}$, i.e., the same order of magnitude as the average current measured in Ref. 1 . The fact that this is not what is seen in the experiment is a major, unresolved puzzle.

Finally, in the experiment of Mailly, Chapelier, and Benoit, ${ }^{3}$ the persistent current has been observed in a GaAs- $\mathrm{Al}_{x} \mathrm{Ga}_{1-x}$ As single loop to have period $\Phi_{0}$ and magnitude $\sim I_{B}$. This result is not too surprising, in view of the fact that the samples are very weakly disordered, and essentially in the ballistic regime, $l / L \sim 1.3$ (one may say that the Thouless energy is replaced here by $\left.\hbar v_{F} / L\right)$. The Coulomb interaction plays no role in this regime, since, in the rotationally invariant limit, Coulomb scattering cannot change the total angular momentum and hence the current.

In this paper, I focus on the theoretical problems posed by the experiments of Ref. 1, which must be described in terms of a disorder average. For three-dimensional, strongly diffusive rings I want to demonstrate (i) that the average persistent current is primarily a Coulomb interaction effect, which is caused by the appearance of a velocity-odd component in the effective one-electron selfenergy, and (ii) that the description of the phenomenon in terms of a "grand canonical" ensemble (more precisely defined below) is justified.

As an introduction to the problem, consider the simplest possible model, namely, a gas of $N$ noninteracting electrons confined by a ring-shaped background of posi- 
tive charge density $n_{+}=N / V$ ( $V$ is the volume of the ring), threaded by a magnetic flux $\Phi$. We disregard the curvature of the ring, and replace it by a linear strip of length $L$ parallel to the $x$ axis, in which the edges at $x=0$ and $x=L$ are identified. The effect of the magnetic flux is completely described by a change in the boundary conditions on the electron wave function:

$$
\psi(x+L, y, z)=\exp [2 \pi i \phi] \psi(x, y, z),
$$

where we have introduced the reduced flux $\phi=\Phi / \Phi_{0}$. The free-electron eigenfunctions satisfying Eq. (1) and vanishing at the edges of the ring are

$$
\psi_{n, k_{\perp}}(x, y, z)=\exp \left[\frac{2 \pi i}{L}(n+\phi) x\right] \sin \left[\mathbf{k}_{\perp} \cdot \mathbf{r}_{1}\right],
$$

where $n$ is a positive or negative integer, and $\mathbf{r}_{1}=y \hat{\mathbf{y}}+z \hat{\mathbf{z}}$ and $k_{\perp}=\left[2 \pi\left(n_{y} \hat{\mathbf{y}}+n_{z} \hat{\mathbf{z}}\right) / L_{\perp}\right]$ are the transverse position vector and wave vector respectively, with $n_{y}$ and $n_{z}$ integers. $L_{\perp}$ is the transverse size of the ring. The eigenvalues are

$$
\epsilon^{(0)}\left(k_{\perp}, n+\phi\right)=\epsilon_{0}(n+\phi)^{2}+\epsilon_{\perp}\left(n_{y}^{2}+n_{z}^{2}\right),
$$

where $\epsilon_{0}=h^{2} / 2 m L^{2}$ and $\epsilon_{\perp}=h^{2} / 2 m L_{1}^{2} \gg \epsilon_{0}$. Each state carries in the $x$ direction a current

$$
I_{n, k_{\perp}}=-\frac{e h}{m L^{2}}(n+\phi),
$$

where $e$ is the absolute value of the electron charge. The total persistent current is calculated by summing the currents carried by the $N$ lowest-lying states. Assume, for simplicity, that only states with $k_{\perp}=0$ are occupied, i.e., the ring is strictly one dimensional. It is simpler at first to calculate the total current carried by the states whose energy is lower than a given chemical potential $\mu$. This is given $b^{5}$

$$
I=\frac{2 I_{B}}{\pi} \sum_{p=1}^{\infty} \frac{\cos \left(p k_{F} L\right)}{p} \sin (2 \pi p \phi),
$$

where $k_{F}$ is the Fermi wave vector, related to the chemical potential by the relation $k_{F}=(2 m \mu)^{1 / 2} / \hbar$, and $v_{F}=\hbar k_{F} / m$ is the Fermi velocity. The sum runs over positive integers $p$. Equation (5) is a periodic function of $\Phi$, with period $\Phi_{0}$, as expected from the periodicity of the boundary condition (1). Its order of magnitude is set by the ballistic current $I_{B}$. Inclusion of the transverse channels of motion changes this result to

$I=-\frac{3 I_{B}}{\pi^{2}}\left(k_{F} L_{\perp}\right) \frac{L_{\perp}}{L} \sum_{p=1}^{\infty} \frac{\sin \left(p k_{F} L\right)}{p^{2}} \sin (2 \pi p \phi)$.

The numerical factor $k_{F} L_{\perp}$ is proportional to the square root of the number of transverse channels of motion, $M \sim 10^{4}$ for the rings used in the experiment of Ref. 1 . $L_{\perp} / L$ is typically a number of order $0.01-0.1$.

The number of electrons in the ring can be fixed by appropriately choosing the chemical potential. From the energy-level diagram shown in Fig. 1 we see that, in the strictly one-dimensional case, this can be achieved with a flux-independent chemical potential $\mu=\epsilon_{0}(N / 2)^{2}$ for

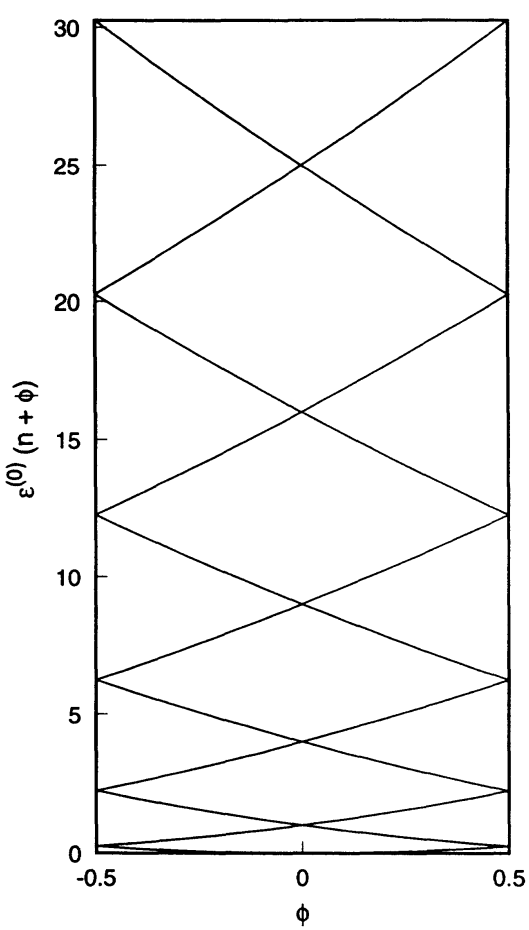

FIG. 1. The energy levels of a strictly one-dimensional ring $\epsilon^{(0)}(n+\phi)=(n+\phi)^{2}$ (in units of $\epsilon_{0}=h^{2} / 2 m L^{2}$ ) are plotted as functions of dimensionless Aharonov-Bohm flux $\phi$, for $n$ in the interval $[-5,5]$ and $\phi$ in the interval $\left[-\frac{1}{2}, \frac{1}{2}\right]$. The number of electrons can be fixed to a value $N$ independent of flux, by choosing the chemical potential $\mu$ equal to one of the zero-flux eigenvalues ( $N$ even) or to one of the $\frac{1}{2}$-flux eigenvalues ( $N$ odd).

even $N$ and $\mu=\epsilon_{0}(N-1 / 2)^{2}$ for odd $N$. Correspondingly, $k_{F} L=\pi N$ for even $N$ and $k_{F} L=2 \pi(N-1 / 2)$ for odd $N$. Then the odd harmonic components of $I$ have positive sign if $N$ is even, or negative sign if $N$ is odd, while the even harmonics are positive, irrespective of the parity of $N$. To compare to experiments, done on a large number of rings $\left[\sim 10^{7}\right.$ (Ref. 1)] containing slightly different numbers of electrons, one needs to take the average between the even- and the odd- $N$ results. Thus one finds

$$
\bar{I}=\frac{I_{B}}{\pi} \sum_{p=1}^{\infty} \frac{1}{p} \sin (4 \pi p \phi)
$$

a periodic function of $\Phi$, with period $\Phi_{0} / 2$. This interesting feature is indeed observed in the experiment of Ref. 1, but the measured current appears to be closer in magnitude to the "diffusive current" $I_{D}=I_{B} l / L$. We now turn to the discussion of this problem.

Because each ring in the ensemble has a slightly different configuration of impurities, a random electronimpurity potential must be included, and the current must be averaged with respect to the impurity distribution. A rigorous treatment of the problem, within the grand canonical ensemble, would require that we assign a different chemical potential to each ring, depending on the number of electrons and the impurity distribution in 
that ring. Working with a fluctuating chemical potential is mathematically inconvenient. Therefore the problem has been divided into two steps. In the first step one neglects the fluctuations of the chemical potential, by assuming that all the rings have the same chemical potential $\mu$. This approximate description has been dubbed the "grand canonical" ensemble in the literature. In the second step, the additional contribution of fluctuations in the chemical potential is expressed in terms of quantities that can be calculated at fixed chemical potential, using an elegant procedure introduced by Altshuler, Gefen, and Imry.

Let us first summarize the results that have been obtained for the case of noninteracting electrons. In the "grand canonical" ensemble one finds (see Ref. 12 and the next section) that the $p$ th harmonic component of the current is damped by an exponential factor $\exp [-p L / 2 l]$. Since $L / l \sim 100$ in typical rings, even the first harmonic of the current should be practically unobservable. Taking into account the fluctuations of the chemical potential from ring to ring leads to a finite average current, whose period is $\Phi_{0} / 2$ and whose magnitude is $^{7,8} \sim I_{B} / M$, where $M \sim\left(k_{F} L_{\perp}\right)^{2}$ is the number of transverse channels of motion. Since $M \sim 10^{4}$ for the threedimensional rings used in the experiment of Ref. 1, the predicted current is still considerably smaller than the measured one $\left(I_{D} \sim 0.01 I_{B}\right)$. More importantly, the theoretical result scales as the inverse of the volume of the ring, whereas, from the physical arguments given above, one would expect a result of the order of the diffusion current. Thus the noninteracting model does not provide a satisfactory answer to the question of the origin and magnitude of the average persistent current in three-dimensional rings.

The outcome of the noninteracting theories indicates that the Coulomb interaction between the electrons should be seriously considered as a possible mechanism for persistent currents. The role of the Coulomb interaction in the persistent-current problem has been recently studied by several authors. ${ }^{9-11}$ The main idea has been to identify a flux-dependent contribution to the average thermodynamic potential $\bar{\Omega}$. According to thermodynamics, the persistent current (at fixed chemical potential) is then given by

$$
I=-c \frac{\partial \bar{\Omega}(\mu, T, \Phi)}{\partial \Phi} .
$$

The novel feature of the interacting theory is that the Coulomb interaction opens up new channels of disorderinduced correlation between the electrons, such as the "cooperon" and the "diffuson." 14 The Cooper correlation, in particular, is sensitive to the magnetic flux, as one knows from the theory of weak localization. In Ref. 9, Ambegaokar and Eckern (AE) calculated the diagrams for $\bar{\Omega}$ to the first order in the screened Coulomb interaction. By letting two density fluctuation loops interact with each other via a cooperon-enhanced screened Coulomb interaction, they obtained an average persistent current of the order of $I_{D}$. Although there remained considerable uncertainty in the value of the proportionality constant, this work provided strong evidence that the
Coulomb interaction could be the primary cause of persistent currents. It must be noted that the AE calculation was done in the "grand canonical" ensemble. We shall show, later, that corrections due to fluctuations in chemical potential from ring to ring are indeed rather small.

Although the Cooper correlator provides a mathematically appealing mechanism for persistent currents, the physical content of the theory remains somewhat obscure. In particular, the following puzzle remains. Suppose that the Coulomb interaction is treated within the Hartree approximation, i.e., we consider each electron moving in the self-consistent electrostatic field created by the electronic charge distribution, plus the external impurity potential. It would appear that such a problem is formally similar to that of noninteracting electrons in the presence of an external random potential. But this latter problem is known to yield an exponentially small average current in the "grand canonical" ensemble, in the diffusive regime. Clearly, there must be a subtle difference between the self-consistent effective potential which appears in the Hartree approximation, and a genuinely external random potential. What is the nature of this difference?

To answer this question, we must understand in detail the mechanism that leads to the exponential vanishing of the "grand canonical" noninteracting current. The average persistent current, like any other single-particle property, can be expressed exactly in terms of the average one-electron Green's function. The average Green's function is, in turn, expressed in terms of an effective self-energy function which controls the position of its poles - the "quasiparticle" energies - in the complex plane. In the noninteracting system, the self-energy is an even function of the electron velocity. It is this one mathematical property that causes the exponential vanishing of the persistent current.

The inclusion of the Coulomb interaction, even at the level of the Hartree approximation, suffices to produce a component of the self-energy which is an odd function of the electron velocity. Although this component is very small (in fact, it vanishes in the macroscopic limit), it breaks the delicate balance of positive and negative contributions that yielded an exponentially small current in the noninteracting case.

The basic physical effect responsible for the velocityodd self-energy is that the electrostatic repulsion between two free-electron states is, on the average, enhanced when the two states have almost opposite velocities. These are the pairs of states which are most strongly correlated by the cooperon. The magnetic flux breaks the symmetry between the average occupation of positive- and negative-velocity states. This broken symmetry is not by itself sufficient to yield a persistent current in the diffusive regime (while it could in the ballistic regime). It is, nevertheless, capable of creating an asymmetry in the electrostatic component of the self-energy as a function of velocity. It is the asymmetry in the functional dependence of the self-energy on velocity-and not just the trivial asymmetry of the eigenvalues of the velocity - that ultimately leads to a finite persistent current.

This paper is organized as follows. In Sec. II, it is 
shown that the effective self-energy function can be written as the sum of a "velocity-even" term, which is an even function of both velocity and magnetic flux, and a "velocity-odd" term, which is an odd function of both these variables. It is then shown that the existence of a finite average persistent current, in the diffusive regime, in the "grand canonical" ensemble, requires the presence of a velocity-odd component of the self-energy. This last result does not depend on the specifics of any microscopic model, but relies on a very general assumption about the existence of quasiparticle poles of the Green's function.

Specific mechanisms are considered beginning in Sec. III. There, we show that impurity scattering yields a purely velocity-even self-energy in the self-consistent Born approximation, and presumably also in an exact treatment. Since the velocity-odd component of the selfenergy is absent, the average persistent current vanishes in the noninteracting theory.

In Sec. IV we calculate the Coulomb contribution to the effective self-energy to first order in the screened Coulomb interaction. We show that the Coulomb selfenergy does have a velocity-odd component, and we use it to compute the average persistent current, which is found to agree with the result of Ambegaokar and Eckern.

In Sec. V, a phenomenological model is introduced, in which each electron is assumed to interact exclusively with a time-reversed partner. This model can be viewed as an extreme idealization of the cooperon physics. Whereas the cooperon enhances the Coulomb interaction between one-electron states whose velocities $v_{n}$ and $v_{n^{\prime}}$ satisfy the condition $\left|v_{n}+v_{n^{\prime}}\right|<(\hbar / m L)\left(E_{c} \tau\right)\left(\tau=l / v_{F}\right.$ is the mean time of flight between elastic collisions), the proposed model reduces the range of interaction to $\left|v_{n}+v_{n^{\prime}}\right| \sim 0$. We show that this model yields the essential qualitative features of the persistent current at $T=0$, namely, the order of magnitude and the half-flux periodicity. More subtle features, such as the temperature dependence and the harmonic content, are not correctly described.

Finally, Sec. VI addresses the problem of the fluctuations of the chemical potential from ring to ring, in the presence of Coulomb interactions. We use the method introduced in Ref. 7 to calculate the change in the average current due to these fluctuations. The result is that the Coulomb corrections to the noninteracting expression cancel out exactly. The change in current has thus the same value as in the noninteracting theory of Ref. 7, and it is small compared to the current computed in the interacting "grand canonical" ensemble.

\section{PERSISTENT CURRENT IN THE GRAND CANONICAL ENSEMBLE}

A ring is described as a bar of length $L$ in the $x$ direction and transverse cross section $L_{1}^{2}$ in the $y-z$ plane, where $x=0$ and $x=L$ are identified. The electrons are subjected to (1) the electrostatic potential of a rigid background of positive charge density $n_{+}$modeling the effect of the crystal lattice (ions + core electrons) on the conduction electrons, (2) a potential $V_{i}(\mathbf{r})$ due to randomly dis- tributed impurities, and (3) a uniform vector potential $\mathbf{A}(\mathbf{r})=\Phi \hat{\mathbf{x}} / L$, modeling the magnetic flux $\Phi$ through the ring. We consider an ensemble of such rings, and assume that they all have the same chemical potential $\mu$. This seemingly unphysical assumption will be discussed in detail in Sec. VI.

The uniform vector potential can be eliminated by a singular gauge transformation, which leads to the "twisted" boundary condition

$$
\Psi(\mathbf{r}+L \hat{\mathbf{x}})=\Psi(\mathbf{r}) \exp [2 \pi i \phi],
$$

for the electron field operator $\Psi(\mathbf{r})$. We shall use this gauge in the rest of this paper. The persistent current, i.e., the amount of charge that flows through an arbitrary cross section of the ring per unit time, is given by

$$
I(\Phi)=\frac{i e \hbar}{m L} \int d \mathbf{r}\left\langle\Psi^{+}(\mathbf{r}) \nabla_{x} \Psi(\mathbf{r})\right\rangle,
$$

where the angular bracket denotes the thermal average in the grand canonical ensemble. An exact representation of Eq. (10) can be given in terms of the temperature Green's function

$G_{\phi}\left(\mathbf{r}, \mathbf{r}^{\prime}, i \omega_{v}\right)=-\int_{0}^{\beta \hbar} d \tau e^{i \omega_{v} \tau}\left\langle\Psi(\mathbf{r}, \tau) \Psi^{+}\left(\mathbf{r}^{\prime}, 0\right)\right\rangle$

where

$$
\Psi(\mathbf{r}, \tau)=\exp (\hat{\mathbf{K}} \tau) \Psi(\mathbf{r}, 0) \exp (-\hat{\mathbf{K}} \tau)
$$

is the imaginary time evolution of the field operator, and $\omega_{v}=2 \pi(\nu+1 / 2) / \beta$ is a fermionic Matsubara frequency, with $\beta=1 / k_{B} T$ and $v$ an integer. The subscript $\phi$ is a reminder of the twisted boundary condition. The persistent current is given by

$I(\Phi)=\frac{i e \hbar}{m L} \int d \mathbf{r} \frac{1}{\beta} \sum_{v} \nabla_{x} G\left(\mathbf{r}, \mathbf{r}^{\prime}, i \omega_{v}\right)_{\mathbf{r}=\mathbf{r}^{\prime}} e^{i \omega_{v} \eta}$

where $\eta \rightarrow 0$.

Equation (12) must be averaged with respect to the impurity distribution. The gradient and integral operations evidently commute with impurity averaging. Ignoring the breaking of translational invariance that occurs near the surface of the rings, the average Green's function depends only on the separation $\mathbf{r}-\mathbf{r}^{\prime}$, and hence it can be written as

$$
\begin{array}{r}
\mathbf{G}_{\phi}\left(\mathbf{r}-\mathbf{r}^{\prime}, i \omega_{v}\right)=\frac{1}{V} \sum_{k_{1}, n} \mathbf{G}_{\phi}\left(k_{\perp}, n+\phi, i \omega_{v}\right) e^{i \mathbf{k}_{\perp} \cdot\left(\mathbf{r}_{\perp}-\mathbf{r}_{\perp}^{\prime}\right)} \\
\times \exp \frac{2 \pi i}{L}(n+\phi)\left(x-x^{\prime}\right),
\end{array}
$$

where $\bar{G}_{\phi}\left(k_{1}, n+\phi, i \omega_{v}\right)$ is a function of flux both implicitly, i.e., through the velocity $\sim n+\phi$, and explicitly, as noted by the $\phi$ subscript. Clearly, this expansion satisfies the boundary conditions. Then the average persistent current, from Eq. (12), can be written as 
$\bar{I}(\Phi)=-\frac{e \hbar}{m L} \sum_{k_{1}, n} \frac{1}{\beta} \sum_{v} \bar{G}_{\phi}\left(k_{1}, n+\phi, i \omega_{v}\right) \frac{2 \pi}{L}(n+\phi) e^{i \omega_{v} \eta}$

It is convenient, at this point, to express the average Green's function in terms of an effective self-energy function $\Sigma$, defined in the following manner:

$$
\begin{aligned}
\bar{G}_{\phi}\left(k_{\perp}, n+\phi, i \omega_{v}\right) & \\
& =\frac{1}{\hbar\left(i \omega_{v}+\mu\right)-\epsilon^{(0)}\left(k_{\perp}, n+\phi\right)-\Sigma_{\phi}\left(k_{\perp}, n+\phi, i \omega_{v}\right)},
\end{aligned}
$$

where $\epsilon^{(0)}$ is the free-electron energy defined in Eq. (3). In a noninteracting system with randomly distributed short-range scatterers, a typical approximation is, ${ }^{15}$

$$
\Sigma_{\phi} \simeq-\frac{i \hbar}{2 \tau} \operatorname{sgn}\left(\omega_{v}\right)
$$

independent of frequency, momentum, and magnetic flux, in a range of frequencies $\left|\omega_{v}\right|<1 / \tau$. In the general case, $\Sigma_{\phi}$ includes contributions from both impurity scattering and Coulomb interaction.

The self-energy determines the position and the width of the quasiparticle peaks in the average spectral function

$$
\bar{A}_{\phi}\left(k_{\perp}, n+\phi, \omega\right)=-\frac{1}{\pi} \operatorname{Im} \bar{G}_{\phi}\left(k_{\perp}, n+\phi, i \omega_{v} \rightarrow \omega+i \eta\right) .
$$

For example, in the noninteracting model with shortrange scatterers, these peaks have a Lorentzian shape of width $\sim \hbar / \tau$ in frequency (or $1 / l$ in wave vector) centered at the free-electron eigenvalues. Because $E_{F} \tau \gg>1$ (and also $k_{F} l \gg 1$ ), the peaks in the spectral function, for $k \sim k_{F}$, sharply define a quasiparticle dispersion in wavevector space, even though, strictly speaking, the wave vector is not a good quantum number. This important feature is expected to persist in the presence of Coulomb interactions.

We now turn our attention to a crucial question, namely the symmetry of the self-energy function. The crucial observation is that the one-electron properties are invari- ant under a simultaneous inversion of the electron velocity and of the magnetic flux. This implies that the selfenergy must have the symmetry

$$
\Sigma_{\phi}\left(k_{\perp}, w_{n}, i \omega_{v}\right)=\Sigma_{-\phi}\left(k_{1},-w_{n}, i \omega_{v}\right),
$$

where $w_{n}=n+\phi$ is the electron velocity, expressed in units of $\hbar / m L$. Notice that the self-energy is defined as a function of flux and velocity, rather than, for example, flux and canonical momentum. This choice of variables is essential to the following development. If $\Sigma$ had no explicit dependence on $\Phi$, Eq. (18) would imply that it must be an even function of velocity. But, in general, the form of the self-energy function compatible with Eq. (18) is

$$
\Sigma_{\phi}\left(k_{\perp}, w_{n}, i \omega_{v}\right)=\Sigma_{\phi}^{(e)}\left(k_{\perp}, w_{n}, i \omega_{v}\right)+\Sigma_{\phi}^{(o)}\left(k_{\perp}, w_{n}, i \omega_{v}\right)
$$

where $\Sigma^{(e)}$ is an even function of the velocity and, independently, of the flux, i.e.,

$$
\begin{aligned}
\Sigma_{\phi}^{(e)}\left(k_{\perp}, w, i \omega_{v}\right) & =\Sigma_{\phi}^{(e)}\left(k_{\perp},-w, i \omega_{v}\right) \\
& =\Sigma_{-\phi}^{(e)}\left(k_{\perp}, w, i \omega_{v}\right) .
\end{aligned}
$$

Similarly $\Sigma^{(o)}$ is an odd function of the velocity and of the magnetic flux:

$$
\begin{aligned}
\Sigma_{\phi}^{(o)}\left(k_{\perp}, w, i \omega_{v}\right) & =-\Sigma_{\phi}^{(o)}\left(k_{\perp},-w, i \omega_{v}\right) \\
& =-\Sigma_{-\phi}^{(o)}\left(k_{\perp}, w, i \omega_{v}\right) .
\end{aligned}
$$

The two components of $\Sigma$ will be referred to as "velocity-even" and "velocity-odd," respectively. Notice that the two functions $\Sigma_{\phi}^{(e)}$ and $\Sigma_{\phi}^{(o)}$ have been analytically continued to arbitrary complex values of the velocity, which are not necessarily physical eigenvalues of the velocity in the ring. Thus, for example, $-w_{n}=-n-\phi$ is not a physical eigenvalue at flux $\Phi$.

We now prove that, if the velocity-odd component of the self-energy is absent, the average persistent current is exponentially small in the diffusive regime. First, we return to Eq. (14) and use standard mathematical techniques to convert the Matsubara sum to an integral of the spectral function over real frequencies,

$$
I(\Phi)=\frac{2 e \hbar^{2}}{m L^{2}} \operatorname{Im} \sum_{k_{\perp}} \int d \omega f(\hbar \omega-\mu) \sum_{n} \frac{n+\phi}{\hbar \omega-\hbar^{2} k_{\perp}^{2} / 2 m-\epsilon_{0}(n+\phi)^{2}-\Sigma_{\phi}^{(e)}\left(k_{\perp}, n+\phi, \omega+i \eta\right)},
$$

where $f(\hbar \omega-\mu)$ is the Fermi distribution function at temperature $T$ and chemical potential $\mu$, and we have assumed that the velocity-odd component of the self-energy is zero. Let us consider a typical value of $\hbar \omega \sim \mu, k_{1} \sim 0$, and let us focus on the sum over $n$,

$$
S=\sum_{n} \frac{n+\phi}{\mu-\epsilon_{0}(n+\phi)^{2}-\Sigma_{\phi}^{(e)}\left(k_{1}, n+\phi, \mu+i \eta\right)} .
$$

If we replaced the discrete sum $\Sigma_{n}$ by a continuous in- tegral $\int d n$ we would obtain trivially zero by symmetry. To refine our estimate, we use the Poisson summation formula

$$
\sum_{n} g(n)=\frac{1}{2 i} \int \operatorname{coth}(\pi z) g(-i z),
$$

in which $g(-i z)$ is an analytic function of $z$, except for a finite number of poles. The contour of integration includes all the poles of $\operatorname{coth}(\pi z)$, but none of the poles of 
$g(-i z)$. Equation (23) is thus rewritten as

$$
S=\frac{1}{2 i} \int \operatorname{coth}(\pi z) \frac{-i z+\phi}{\mu-E(-i z+\phi)} d z,
$$

where we have defined the complex function of the complex dimensionless velocity $w$

$$
E(w)=\epsilon_{0} w^{2}+\Sigma_{\phi}^{(e)}\left(k_{1}, w, \mu+i \eta\right) .
$$

For the sake of simplicity, we have omitted writing the dependence of $E$ on frequency, transverse wave vector, and flux. To go further in the evaluation of Eq. (25), we must make some assumptions about the analytic structure of the integrand. We assume it to be analytic, except for simple poles associated with the zeros of the denominator. Because of the evenness of the function $E(w)$, it is evident that such zeros, if present, must occur in pairs at

$$
z=-i\left(\phi \pm z_{0}\right)
$$

where $\pm z_{0}$ are the two symmetric solutions of the equation

$$
E\left(z_{0}\right)=\mu,
$$

and $z_{0}$ is chosen to have a positive imaginary part.

Are there any solutions to Eq. (28)? In the absence of interactions, with $\Sigma^{(e)} \sim-i \hbar / 2 \tau$ the answer is "yes," with

$$
z_{0}=\left(\frac{\mu+i \hbar / 2 \tau}{\epsilon_{0}}\right)^{1 / 2} \simeq \frac{L}{2 \pi}\left(k_{F}+\frac{i}{2 l}\right)
$$

$\left[k_{F}=(2 m \mu)^{1 / 2} / \hbar\right]$. The physical meaning of this result is that the electronic spectral function, regarded as a function of wave vector, has a Lorentzian peak centered at $k_{F}$ of width $1 / l$ : the spread in wave vector is related to the mean free path by an uncertainty relation. We now assume, in the spirit of Landau's Fermi-liquid theory, that this basic structure of the spectral function is not qualitatively changed by Coulomb interactions. In other words, the solution of Eq. (28), including interactions, is still given by Eq. (29), with a Coulombrenormalized mean free path $l$. We also assume that no other solutions exist, and that $E(w) \rightarrow \epsilon_{0} w^{2}$ for $w \rightarrow \infty$.

Let us return to Eq. (25), and let us distort the contour of integration, as shown in Fig. 2, so as to include the contribution of the poles from Eq. (27). Noting that the contributions of the two half circles at infinity cancel against each other, we obtain the result

$S=\frac{i \pi z_{0}}{E^{\prime}\left(z_{0}\right)}\left\{\operatorname{coth}\left[i \pi\left(z_{0}-\phi\right)\right]-\operatorname{coth}\left[i \pi\left(z_{0}+\phi\right)\right]\right\}$.

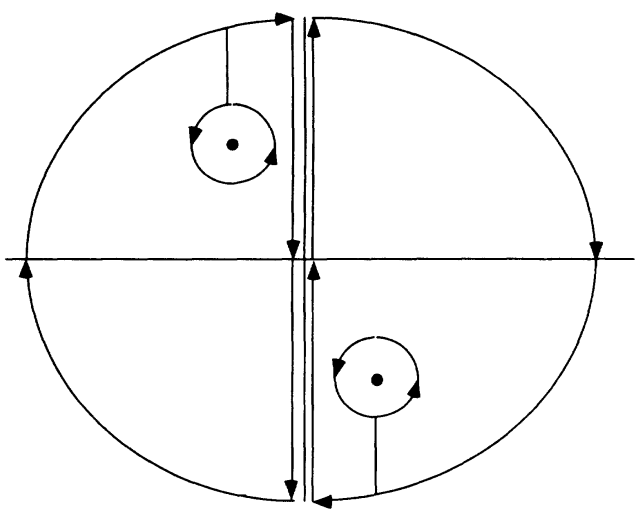

FIG. 2. Contour of integration in the complex $z$ plane, for the evaluation of Eq. (25). The solid dots represent the two poles of Eq. (27).

Now, using Eq. (29) for $z_{0}$, we find

$$
\begin{aligned}
\operatorname{coth}\left[i \pi\left(z_{0}-\phi\right)\right] & =\frac{e^{L / l}-1-2 i e^{-L / 2 l} \sin \left[k_{F} L-2 \pi \phi\right]}{e^{L / l}+1-2 e^{L / 2 l} \cos \left[k_{F} L-2 \pi \phi\right]} \\
& \sim 1+O\left(e^{-L / 2 l}\right) .
\end{aligned}
$$

Substituting this in Eq. (30) we see that the 1 cancels out, the remainder is proportional to the exponential factor $\exp (-L / 2 l)$, which is negligible in the diffusive regime. We have carried out our analysis for the case $\hbar \omega \sim \mu$. It is straightforward to repeat the analysis for the general case $\hbar \omega<\mu$. With the same assumptions about the analytic structure of the integrand, one finds that the damping factor $\exp (-L / 2 l)$ is replaced by an even smaller one, $\exp (-L / 2 l)(\mu / \hbar \omega)^{1 / 2}$. This concludes the proof of our assertion that the average persistent current is exponentially small (in the diffusive regime) if the velocityodd self-energy is absent.

Let us now consider the effect of a small velocity-odd component of the self-energy. It is now permissible to replace in leading order the sum over $n$ by a continuous integral over $k_{x}=2 \pi(n+\phi) / L$, and we obtain a finite $S$ and hence a finite persistent current. Since the persistent current is a mesoscopic effect (i.e., it vanishes in the limit $L \rightarrow \infty$ ) it follows that the velocity-odd component of the self-energy must also be mesoscopic, i.e., of order $1 / L$ at most. It is then justified to treat $\Sigma^{(o)}$ as a small correction to the velocity-even self-energy, since the latter remains finite in the macroscopic limit. Expanding Eq. (22) to first order in $\Sigma^{(o)}$ we obtain the following approximate expression for the current:

$$
I(\Phi)=\frac{e \hbar^{2}}{m} \operatorname{Im} \sum_{k_{\perp}} \int \frac{d \omega}{\pi} f(\hbar \omega-\mu) \int \frac{d k_{x}}{2 \pi} \frac{k_{x} \Sigma_{\phi}^{(o)}\left(k_{1}, k_{x}, \omega+i \eta\right)}{\left[\hbar \omega-\hbar^{2}\left(k_{1}^{2}+k_{x}^{2}\right) / 2 m-\Sigma_{\phi}^{(e)}\left(k_{\perp}, k_{x}, \omega+i \eta\right)\right]^{2}}
$$

This important result shows that the mesoscopic persistent current is directly related to the velocity-odd component of the self-energy.

In physical terms, the presence of a velocity-odd self- energy implies that the kinetic energies, and hence the velocities of left- and right-moving electrons at the top of the Fermi distribution, must differ, to compensate for the asymmetry of the interaction energy. Otherwise, elec- 
trons would be transferred from the right-moving to the left-moving field. Thus the driving force of the persistent current is the asymmetry of the quasiparticle energy as a function of velocity. The asymmetry in the eigenvalue spectrum of the velocity (caused by the magnetic flux) acts only as a "trigger" for this effect.

The important aspect of this result is its generality. Only the symmetry of the Hamiltonian and the physical assumption of the existence of diffusing quasiparticles with mean free path $l$ have been used in deriving it.

\section{NONINTERACTING ELECTRONS IN THE GRAND CANONICAL ENSEMBLE}

In this section, we discuss whether noninteracting electrons can sustain a velocity-odd self-energy - and hence a persistent current-in mesoscopic rings. In the selfconsistent Born approximation (SCBA), the electron self-energy, caused by impurity scattering, is given by the diagram of Fig. 3, in which the thick line represents the self-consistent average Green's function. Its analytic expression is

$$
\begin{aligned}
& \Sigma_{\phi}\left(k_{\perp}, n+\phi, \omega\right) \\
&=n_{i} \sum_{k_{1}^{\prime}, n^{\prime}} \frac{\left|v\left[k_{\perp}-k_{1}^{\prime},(2 \pi / L)\left(n+\phi-n^{\prime}-\phi\right)\right]\right|^{2}}{\hbar \omega-\epsilon^{(0)}\left(k_{\perp}^{\prime}, n^{\prime}+\phi\right)-\Sigma_{\phi^{\prime}}\left(k_{\perp}^{\prime}, n^{\prime}+\phi, \omega\right)},
\end{aligned}
$$

where $v(\mathbf{k}) \cong v\left(k_{1}, k_{x}\right)$ is the Fourier transform of the electron-impurity potential, and $n_{i}$ is the concentration of impurities. We decompose the self-energy into velocityeven and velocity-odd components, and make use of the fact that the odd component is a mesoscopic effect of order $1 / L$, much smaller than the even component. This enables us to decouple the even- and odd-velocity channels up to corrections of higher order in $\Sigma^{(o)}$.

It is convenient to define even and odd components of the squared electron-impurity potential as follows:

$$
\begin{aligned}
\left|v^{(e)}\right|^{2}=\frac{1}{2} & {\left.\left[|v| k_{\perp}-k_{\perp}^{\prime}, \frac{2 \pi}{L}\left(n+\phi-n^{\prime}-\phi\right)\right]\right|^{2} } \\
& \left.+\left|v\left[k_{\perp}-k_{\perp}^{\prime}, \frac{2 \pi}{L}\left(-n-\phi-n^{\prime}-\phi\right)\right]\right|^{2}\right],
\end{aligned}
$$

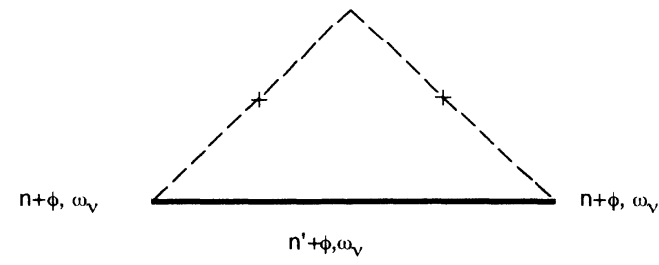

FIG. 3. Diagram for the effective self-energy in the selfconsistent Born approximation for noninteracting electrons. The thick solid line represents the self-consistent average Green's function, and the dashed lines with crosses the average impurity scattering. and

$$
\begin{aligned}
\left|v^{(o)}\right|^{2}=\frac{1}{2} & {\left.\left[|v| k_{\perp}-k_{\perp}^{\prime}, \frac{2 \pi}{L}\left(n+\phi-n^{\prime}-\phi\right)\right]\right|^{2} } \\
& \left.-\left|v\left[k_{\perp}-k_{\perp}^{\prime}, \frac{2 \pi}{L}\left(-n-\phi-n^{\prime}-\phi\right)\right]\right|^{2}\right] .
\end{aligned}
$$

Then we can write

$$
\begin{aligned}
& \Sigma_{\phi}^{(e)}\left(k_{\perp}, n+\phi, \omega\right) \\
& \quad \simeq n_{i} \sum_{k_{1}^{\prime}, n^{\prime}} \frac{\left|v^{(e)}\right|^{2}}{\hbar \omega-\epsilon^{(0)}\left(k_{\perp}^{\prime}, n^{\prime}+\phi\right)-\Sigma_{\phi}^{(e)}\left(k_{\perp}^{\prime}, n^{\prime}+\phi\right)}
\end{aligned}
$$

and

$$
\begin{aligned}
& \Sigma_{\phi}^{(o)}\left(\mathbf{k}_{1}, n+\phi, \omega\right) \\
& \quad \simeq n_{i} \sum_{k_{1}^{\prime}, n^{\prime}} \frac{\left|v^{(o)}\right|^{2}}{\hbar \omega-\epsilon^{(0)}\left(k_{1}^{\prime}, n^{\prime}+\phi\right)-\Sigma_{\phi}^{(e)}\left(\mathbf{k}_{1}^{\prime}, n^{\prime}+\phi\right)} .
\end{aligned}
$$

Note that $v^{(o)}$ and hence $\Sigma^{(o)}$ vanish exactly if $v$ is assumed to be independent of wave vector, i.e., in the case of $\delta$-function impurities. In the general case of finiterange impurities we proceed as follows. First, in Eq. (36), we can take the macroscopic limit, and obtain the well-known solution $^{15}$

$$
\Sigma_{\phi}^{(e)}\left(\mathbf{k}_{\perp}, n+\phi, \omega+i \eta\right) \sim-\frac{i \hbar}{2 \tau},
$$

where $1 / \tau$ is the usual Fermi-surface average ${ }^{15}$ of the electron-impurity scattering probability.

We substitute this result in the equation for $\Sigma^{(o)}$, and observe that the sum on the right-hand side has the same structure as the sum considered in the previous section, Eq. (23). We can analyze it in precisely the same wayby converting the sum to a contour integral-assuming that the potential is well represented by an analytic function of wave vector. The only difference is that the factor $-i z+\phi$ in the numerator of Eq. (23) is replaced by $\left|v^{(o)}\right|^{2}$ of Eq. (35) with $n^{\prime} \rightarrow-i z$. This is still an antisymmetric function of $-i z+\phi$. Thus the result of the wave-vector sum in Eq. (35) is still in the form of Eq. (30) apart from a different prefactor, which reflects a different value of the residue at the pole. Clearly, then, the sum is exponentially small, with an exponential factor of the order of $\exp (-L / 2 l)$.

Thus we have proved that impurity scattering, at least when treated at the level of the SCBA, can only give an exponentially vanishing velocity-odd component of the self-energy. Hence, persistent currents cannot occur in the presence of diffusive impurity scattering alone, when the Coulomb interaction is neglected. We emphasize that this conclusion is valid for the grand canonical ensemble. At this point, we cannot absolutely rule out the possibility that very strong impurity scattering, requiring a treatment beyond the SCBA, could result in a finite velocityodd component of $\Sigma_{\phi}$. However, the presence of such strong impurity effects would be inconsistent with the generally accepted notion that the mesoscopic rings are 
well within the (three-dimensional) weak-localization regime.

\section{INTERACTING ELECTRONS IN THE GRAND CANONICAL ENSEMBLE}

Let us now turn to consideration of electron-electron interactions as a possible mechanism for persistent currents in mesoscopic rings. According to the results of the previous sections, the problem is to understand if and how a velocity-odd component of the one-electron selfenergy can arise from the Coulomb interaction.

First of all, we wish to demonstrate that all the results of the recent theory of Ambegaokar and Eckern ${ }^{9}$ can be obtained from a velocity-odd component of the selfenergy. This analysis provides a microscopic basis for the idealizations introduced in the next section.

We consider the diagrams for the self-energy of first order in the screened Coulomb interaction. They are shown in Fig. 4. The solid lines represent average noninteracting Green's functions. The wavy line represents the screened Coulomb interaction. Following Ambegaokar and Eckern, we approximate the screened Coulomb scattering amplitude as a momentum- and frequencyindependent constant $\bar{V}$. The ladders of impurity lines in the particle-particle channel (the cooperon) describe a disorder-induced correlation between states close to the Fermi energy. The mathematical expression for the cooperon is

$$
K\left(\mathbf{k},-\mathbf{k}+\mathbf{q}, i \omega_{v}, i \omega_{v}-i \omega_{\mu}\right)=\frac{\hbar}{2 \pi N(0) \tau^{2}} \frac{1}{\left|\omega_{\mu}\right|+D q^{2}},
$$

if the Matsubara frequencies $\omega_{v}$ and $\omega_{v}-\omega_{\mu}$ have opposite signs, and essentially zero otherwise. The definition of the wave-vector and frequency labels is shown in Fig. 4. $N(0)$ is the density of states at the Fermi surface, and $D$ is the diffusion constant. The physical effect of the disorder-induced correlation is that an electron interacts more strongly with other electrons of similar energy, but traveling on approximately time-reversed paths. This happens because two electrons on time-reversed paths experience the same impurity distribution, and hence have maxima and minima in charge distribution at approximately the same positions.

Let us evaluate explicitly the self-energy from the diagram of Fig. 4. Dropping for convenience the cumbersome quantum numbers of transverse motion, and combining the Hartree and Fock contributions in a single expression, we obtain

$$
\begin{aligned}
\Sigma_{\phi}\left(n+\phi, i \omega_{v}\right)=\bar{V} \frac{1}{\beta} \sum_{\mu} \sum_{m^{\prime}, m^{\prime \prime}, q} & \bar{G}_{0}\left(-m^{\prime}+q+\phi, i \omega_{v}-i \omega_{\mu}\right) \bar{G}_{0}\left(m^{\prime}+\phi, i \omega_{v}\right) \bar{G}_{0}\left(-m^{\prime \prime}+q+\phi, i \omega_{v}-i \omega_{\mu}\right) \\
& \times \bar{G}_{0}\left(m^{\prime \prime}+\phi, i \omega_{v}\right)\left(\frac{1}{2 \pi N(0) \tau^{2}}\right)^{2}\left(\frac{1}{\left|\omega_{v}\right|+\bar{D}(q+2 \phi)^{2}}\right)^{2} \\
& \times \bar{G}_{0}\left(-n+q+\phi, i \omega_{v}-i \omega_{\mu}\right),
\end{aligned}
$$
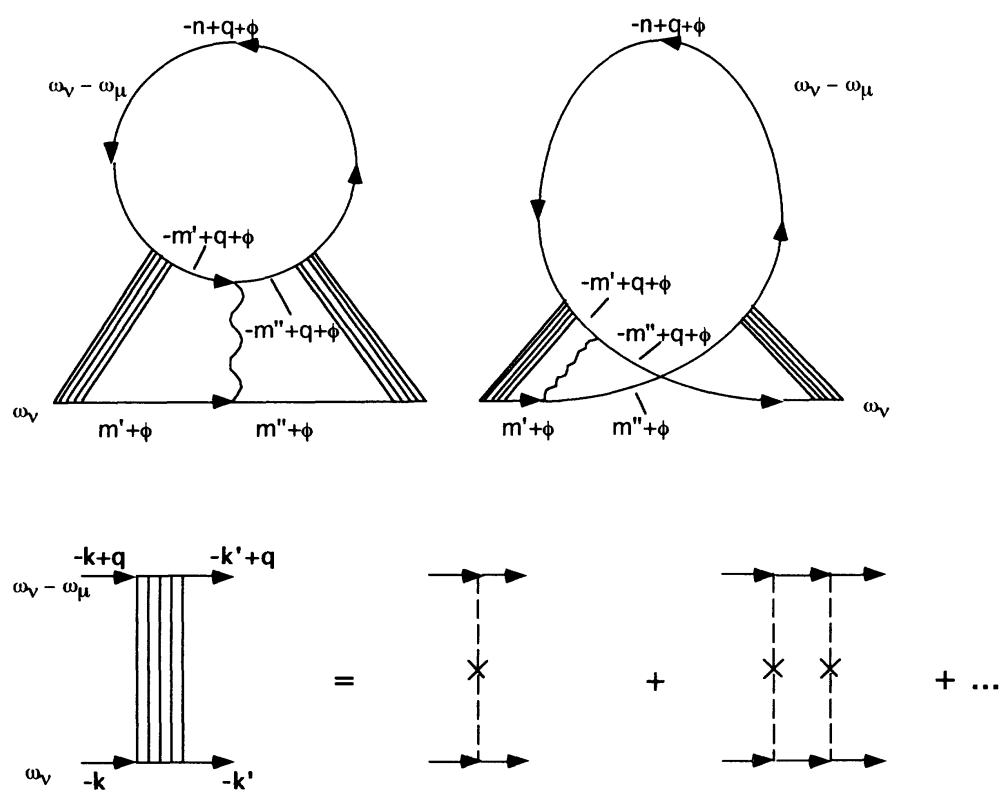

FIG. 4. First-order diagrams for the effective Coulomb selfenergy. The wavy line represents the screened Coulomb interaction, the dashed lines with crosses the average impurity scattering. 
where the sum over Matsubara frequencies is restricted in such a way that $\omega_{v}$ and $\omega_{v}-\omega_{\mu}$ have opposite signs. We have defined $\bar{D}=D(2 \pi / L)^{2}$, and $q$ is now an integer. The sums over integers $m^{\prime}$ and $m^{\prime \prime}$ are done trivially, after setting $q=0$, using the result

$$
\sum_{p} \bar{G}_{0}\left(p, i \omega_{v}-i \omega_{\mu}\right) \bar{G}_{0}\left(p, i \omega_{v}\right)=\frac{2 \pi N(0) \tau / \hbar}{\left|\omega_{\mu}\right| \tau+1} \Theta_{<}\left(\omega_{v}\left(\omega_{v}-\omega_{\mu}\right)\right)
$$

$\left[\Theta_{<}(x)=1\right.$ if $x<0$ and zero otherwise $]$. Then we obtain

$$
\Sigma_{\phi}\left(n+\phi, i \omega_{v}\right)=\bar{V} \frac{1}{\beta \tau^{2}} \sum_{\mu} \sum_{q} \bar{G}_{0}\left(-(n+\phi)+(q+2 \phi), i \omega_{v}-i \omega_{\mu}\right)\left(\frac{1}{\left|\omega_{\mu}\right| \tau+1}\right)^{2}\left(\frac{1}{\left|\omega_{\mu}\right|+\bar{D}(q+2 \phi)^{2}}\right)^{2} .
$$

We now extract the velocity-odd component of Eq. (41) by antisymmetrizing with respect to $n+\phi$ and using a firstorder Taylor expansion for $\bar{G}_{0}$. This yields

$$
\Sigma_{\phi}^{(o)}\left(n+\phi, i \omega_{v}\right)=-2 \epsilon_{0} \frac{\bar{V}}{\beta \tau^{2}} \sum_{\mu} \bar{G}_{0}^{2}\left(n+\phi ; i \omega_{v}-i \omega_{\mu}\right)(n+\phi)\left(\frac{1}{\left|\omega_{v}\right| \tau+1}\right)^{2} \sum_{q} \frac{q+2 \phi}{\left[\left|\omega_{\mu}\right|+\bar{D}(q+2 \phi)^{2}\right]^{2}}
$$

Note that the explicit flux dependence of $\Sigma^{(o)}$ has a period of $\Phi_{0} / 2$ as expected. Now, inserting Eq. (42) into Eq. (32) we get the persistent current

$$
\begin{aligned}
I(\phi)=-\frac{e h}{m L^{2}} \frac{\bar{V}}{4 D \tau^{2}} \sum_{k_{1}, k_{x}} \frac{1}{\beta^{2}} \sum_{\mu} \sum_{v} \bar{G}_{0}^{2}\left(k_{x}, i \omega_{v}-i \omega_{\mu}\right) & \\
& \times \bar{G}_{0}^{2}\left(k_{x}, i \omega_{v}\right) \frac{\hbar^{2} k_{x}^{2}}{m}\left|\frac{1}{\left|\omega_{\mu}\right| \tau+1}\right|^{2} \frac{\partial}{\partial \Phi} \sum_{q} \frac{1}{\left|\omega_{\mu}\right|+\bar{D}(q+2 \phi)^{2}} .
\end{aligned}
$$

Using the standard method to evaluate the wave-vector sum, we obtain

$$
\begin{aligned}
\sum_{\mathbf{k}} \bar{G}_{0}^{2}\left(\mathbf{k}, i \omega_{v}\right. & \left.-i \omega_{\mu}\right) \bar{G}_{0}^{2}\left(\mathbf{k}, i \omega_{v}\right) k_{x}^{2} \\
& \sim \frac{4}{3} \pi N(0) k_{F}^{2} \tau^{3} \hbar^{-3} \Theta_{<}\left(\omega_{v}\left(\omega_{v}-\omega_{\mu}\right)\right) .
\end{aligned}
$$

The final expression for the current is

$$
\begin{aligned}
I(\Phi)=- & c N(0) \bar{V} \frac{\partial}{\partial \Phi} \frac{1}{\beta} \\
& \times \sum_{v} \sum_{q} \frac{\left|\omega_{v}\right|}{\left|\omega_{v}\right|+\bar{D}(q+2 \phi)^{2}}\left(\frac{1}{\left|\omega_{v}\right| \tau+1}\right)^{2} .
\end{aligned}
$$

It coincides with the expression obtained by Ambegaokar and Eckern ${ }^{9}$ from taking the flux derivative of the thermodynamic potential. The harmonic content of the average current is (if $E_{c} \tau<<1$ ) (Refs. 9,12)

$$
I(\Phi)=\sum_{p=1}^{\infty} I_{p} \sin (4 \pi p \phi)
$$

where

$$
I_{p}=\frac{e}{h} N(0) \bar{V} 4 \pi p E_{c} \bar{T} \sum_{\mu>0} \bar{\omega}_{\mu}^{1 / 2} e^{-p \bar{\omega}_{\mu}^{1 / 2}},
$$

where $\bar{\omega}_{\mu}=2 \pi \mu \bar{T}$ is the Matsubara frequency associated with the dimensionless temperature $\bar{T}=k_{B} T / E_{c}$, and $E_{c}=\hbar D / L^{2}$ is the Thouless energy.

The point of this alternative derivation is that it illustrates the essential role played by the velocity-odd selfenergy, i.e., the asymmetric quasiparticle dispersion, in causing the persistent current. The persistent current arises solely from the velocity-odd part of the Coulomb self-energy. Two electrons moving with opposite velocities have their energies shifted by opposite amounts, leading to an asymmetry in the velocity distribution which is robust with respect to diffusive impurity scattering.

\section{A SIMPLE MODEL FOR PERSISTENT CURRENTS}

The analysis of the Ambegaokar-Eckern theory in the previous section suggested that the key physical effect in the phenomenon of persistent current is the enhancement of the electrostatic repulsion between states with almost opposite velocities. More precisely, from the analytical expression of the cooperon it follows that pairs of states whose dimensionless velocities $w_{n}, w_{n^{\prime}}$ satisfy the condition $\left|w_{n}+w_{n^{\prime}}\right|<L / \hbar$ interact more strongly than average. The factor by which the Coulomb interaction between the states $w_{n}$ and $w_{n}$, is enhanced is approximately proportional to

$$
\frac{1}{\beta} \sum_{v} \frac{1}{\hbar\left|\omega_{v}\right|+E_{c}\left|w_{n}+w_{n^{\prime}}\right|^{2}},
$$

where the sum over Matsubara frequencies is cut off at a value of order $1 / \tau$.

The Lorentzian form of the summand, regarded as a function of the center-of-mass velocity, suggests that we consider an idealized model of the electron-electron interaction, in which an electron in a state of velocity $w_{n}$ interacts exclusively with the state of velocity $w_{\bar{n}}$ which is closest to having exactly the opposite velocity, i.e., it is 
such that

$$
\left|w_{n}+w_{\bar{n}}\right|=|n+\bar{n}+2 \phi|
$$

is minimum. The state $\bar{n}$ defined by this condition will be referred to as the time-reversed partner of state $n$. Clearly, one has

$$
w_{\bar{n}}=-w_{n}+2 \phi-[2 \phi],
$$

where $[x]$ denotes the integer which is nearest to $x$.

Let $\bar{V}$ denote the matrix element of the screened Coulomb interaction between $w_{n}$ and $w_{\bar{n}}$. Then the firstorder Coulomb self-energy is given by

$$
\Sigma_{\phi}^{\operatorname{Coul}}\left(k_{\perp}, w_{n}\right)=\bar{V} f\left(\epsilon_{0} w_{\bar{n}}^{2}+\epsilon_{k_{\perp}}-\mu\right),
$$

i.e., the screened Coulomb interaction times the thermal occupation factor of the time-reversed partner.

Next, we extract the velocity-odd component of the self-energy, by antisymmetrizing Eq. (51) with respect to $w_{n}$. Using the fact that $n \gg 1$ for states near the Fermi level, we can express the result of the antisymmetrization in terms of a first-order Taylor expansion with respect to $\phi$. Thus we obtain

$$
\begin{array}{r}
\Sigma_{\phi}^{(o)}\left(k_{1}, w_{n}\right)=- \\
2 \bar{V} f^{\prime}\left(\epsilon_{0} w_{n}^{2}+\epsilon_{k_{1}}-\mu\right) \\
\times \epsilon_{0} w_{n}([2 \phi]-2 \phi),
\end{array}
$$

where $f^{\prime}(\epsilon-\mu)$ is the derivative of the Fermi distribution with respect to its argument. We substitute this expression into our general formula for the persistent current Eq. (32). Since we are doing a calculation up to first order in the screened Coulomb interaction, we are entitled to neglect the contribution of the Coulomb interaction to the velocity-even part of the self-energy. The latter is then taken to be of the form of Eq. (16). In the zerotemperature limit, the derivative of the Fermi distribution is a $\delta$ function, and all the integrals in Eq. (32) become elementary. The result for the average persistent current in the three-dimensional case is

$$
I(\Phi)=\frac{4}{3} N(0) \bar{V} I_{D}([2 \phi]-2 \phi)
$$

where $I_{D}$ is the diffusion current, defined in the Introduction. This equation is periodic in $\Phi$, with period $\Phi_{0} / 2$.

The temperature dependence of the persistent current is more problematic. Assuming that only electrons within a range of energies $E_{c}$ around the Fermi energy are affected by the "odd self-energy" effect, we find that the persistent current is independent of temperature for $T<E_{c}$, and has a power-law decay proportional to $E_{c} / T$ for $T>E_{c}$. This behavior is in conflict with the generally accepted result that the persistent current decays exponentially with temperature, ${ }^{13}$ with a characteristic scale $E_{c}$. The origin of the difficulty can be easily traced back to the assumption that the range of cooperon vanishes in velocity space, which amounts to taking the limit $E_{c} \rightarrow \infty$ in Eq. (48).

In conclusion, the major qualitative features of the Ambegaokar-Eckern result - the order of magnitude and the half-flux periodicity of the current-are obtained with remarkable simplicity from our model. More detailed features, such as the exponential temperature dependence and the precise form of the harmonic content of the current, depend on the finite extent of the cooper correlation in velocity space [see Eq. (48)], and cannot be correctly described by this model.

\section{FLUCTUATIONS IN THE CHEMICAL POTENTIAL}

Thus far, we have studied the persistent current in a grand canonical ensemble of rings having a fixed value of the chemical potential. In reality, the chemical potential fluctuates from ring to ring, due to slight differences in geometry, number of electrons, and impurity distribution. A simple way to take into account the effect of these fluctuations in the chemical potential has been recently suggested by Altshuler, Gefen, and Imry. ${ }^{7}$ The essential idea of this approach is to express the physical fluctuation $\delta \mu$ of the chemical potential in terms of a fluctuation $\delta N$ in the number of electrons, calculated at constant $\mu$ :

$$
\delta \mu(\phi) \simeq\left(\frac{\partial N}{\partial \mu}\right)^{-1} \delta N(\phi)
$$

Then it is straightforward to show ${ }^{7}$ that the correction to the "grand canonical" persistent current is given by

$$
\delta I(\phi)=-\frac{c}{2}\left[\frac{\partial N}{\partial \mu}\right]^{-1} \frac{\partial}{\partial \Phi} \overline{[\delta N(\phi)]^{2}}
$$

where $\delta N(\phi)=N(\phi)-\bar{N}$ is the fluctuation in the electron number at fixed chemical potential, $\bar{N}$ is the average number of electrons in a ring (a flux-independent quantity) and the overbar denotes the impurity average. All the quantities appearing on the right-hand side of Eq. (55) can be computed in the grand canonical ensemble, i.e., at fixed chemical potential. This formula is valid for interacting as well as noninteracting electrons. The calculation for noninteracting electrons was done by Altshuler, Gefen, and Imry. ${ }^{7}$ We summarize their results. The variance of the particle number, as a function of flux, is given by the Fourier series

$$
\overline{\delta N(\phi)^{2}}=\frac{2 \bar{T}}{\pi} \sum_{p=1}^{\infty} \cos \left(4 \pi p \phi ; \sum_{v>0} \bar{\omega}_{v}^{-1 / 2} \exp \left(-p \bar{\omega}_{v}^{1 / 2}\right),\right.
$$

where $\bar{T}=k_{B} T / E_{c}$ and $\bar{\omega}_{v}=2 \pi v \bar{T}$. The compressibility factor $(\partial N / \partial \mu)^{-1}$ is approximately equal to the inverse of the density of states at the Fermi surface $\Delta=\pi^{2} \hbar^{2} k_{F} / m L M$, where $M=\left(k_{F} L_{\perp}\right)^{2}$ is the number of transverse channels. This implies that the Fourier components of the persistent current with respect to flux are of the order of the ballistic current divided by the number of transverse channels:

$$
\begin{aligned}
\delta I(\Phi) \cong I(\Phi)=\frac{e \Delta}{h} \frac{4 \bar{T}}{\pi} \sum_{p=1}^{\infty} p & \sin (4 \pi p \phi) \\
& \times \sum_{v>0} \bar{\omega}_{v}^{-1 / 2} \exp \left(-p \bar{\omega}_{v}^{1 / 2}\right) .
\end{aligned}
$$


We have used the fact that the "grand canonical" current is negligibly small for noninteracting electrons. As discussed in the Introduction, this result falls about two orders of magnitude short of explaining the magnitude of the persistent current observed in the experiment of Ref. 1.

In the rest of this section we include the Coulomb interaction in the calculation of $\delta I$, and we show its effect to be negligible. Although the compressibility and the variance of the particle number are quite strongly affected by the Coulomb interaction, their combination in Eq. (55) is not. Hence, $\delta I$ remains small, in the sense explained above.

To calculate the derivative of $\delta N$ with respect to flux in the presence of interaction, we resort to the well-known Kohn-Sham representation of the equilibrium density of a nonuniform many-electron system. According to this theory, ${ }^{16-18}$ the particle number at equilibrium is given by

$$
N=\sum_{\alpha} f\left(\epsilon_{\alpha}-\mu\right)
$$

where $f$ is the Fermi distribution, $\epsilon_{\alpha}$ are the eigenvalues of the self-consistent Kohn-Sham equation

$$
\begin{aligned}
{\left[-\frac{\hbar^{2} \nabla^{2}}{2 m}+V_{i}(\mathbf{r})\right.} & +V_{H}[n(\mathbf{r}), \mathbf{r}] \\
& \left.+V_{\mathrm{XC}}[n(\mathbf{r}), \mathbf{r}]\right] \psi_{\alpha}(\mathbf{r})=\epsilon_{\alpha} \psi_{\alpha}(\mathbf{r}),
\end{aligned}
$$

and $V_{H}[n(\mathbf{r}), \mathbf{r}]$ and $V_{\mathrm{XC}}[n(\mathbf{r}), \mathbf{r}]$ are the Hartree and the exchange-correlation potentials, respectively, functionals of the self-consistent density

$$
n(\mathbf{r})=\sum_{\alpha} f\left(\epsilon_{\alpha}-\mu\right)\left|\psi_{\alpha}(\mathbf{r})\right|^{2}
$$

Taking into account Eqs. (58) and (59) we can write the variation of particle number with respect to flux as follows:

$$
\frac{\partial \delta N(\phi)}{\partial \phi}=\left.\frac{\partial}{\partial \phi} \sum_{\alpha} f\left(\epsilon_{\alpha}-\mu\right)\right|_{s}+\sum_{\alpha} \frac{\partial f\left(\epsilon_{\alpha}-\mu\right)}{\partial \epsilon_{\alpha}} \int d^{3} r \int d^{3} r^{\prime} \frac{\delta \epsilon_{\alpha}}{\delta V_{H \mathrm{XC}}(\mathbf{r})} \frac{\delta V_{H \mathrm{XC}}(\mathbf{r})}{\delta n\left(\mathbf{r}^{\prime}\right)} \frac{\partial n\left(\mathbf{r}^{\prime}\right)}{\partial \phi}
$$

The first term on the right-hand side of this equation describes the variation arising from the change in magnetic flux, at constant effective potentials. The second term takes into account the fact that the effective potentials $V_{H}$ and $V_{\mathrm{XC}}\left(V_{H \mathrm{XC}}=V_{H}+V_{\mathrm{XC}}\right)$ change in response to a change in flux, because they are functionals of the density. Now we observe that the $\mathbf{r}$ dependence of the sum over eigenvalues

$$
\sum_{\alpha} \frac{\partial f\left(\epsilon_{\alpha}-\mu\right)}{\partial \epsilon_{\alpha}} \frac{\delta \epsilon_{\alpha}}{\delta V_{H \times C}(\mathbf{r})}
$$

can be safely neglected in a first approximation, i.e., we can replace this quantity by its average over the volume of the ring. Since Eq. (61) describes the response of the electron number to a scalar potential applied at point $r$, it is evident that its average over the volume can be expressed in terms of the response to a uniform scalar potential, i.e., a change in chemical potential. Thus, the expression appearing in Eq. (61) is replaced by $-(\partial N / \partial \mu) / V \sim-1 / \Delta V$, where $V$ is the volume of the ring and $\Delta$ is the mean separation between energy levels of the Kohn-Sham equation. Similarly, the functional derivative

$$
\frac{\delta V_{H \mathrm{XC}}(\mathbf{r})}{\delta n\left(\mathbf{r}^{\prime}\right)}=\frac{e^{2}}{\left|\mathbf{r}-\mathbf{r}^{\prime}\right|}-f_{\mathrm{XC}}\left(\left|\mathbf{r}-\mathbf{r}^{\prime}\right|\right)
$$

can be treated as a function of $\left|\mathbf{r}-\mathbf{r}^{\prime}\right|$. This function describes the functional dependence of the self-consistent potential on density, the first term representing the classical Hartree contribution and the second term the quantum exchange-correlation effects. Finally, $\partial n\left(\mathbf{r}^{\prime}\right) / \partial \phi$, when integrated over the volume, regenerates the un- known function of $\partial \delta N(\phi) / \partial \phi$. Collecting all these results together, we obtain

$$
\frac{\partial \delta N(\phi)}{\partial \phi}=\left.\frac{\partial \delta N(\phi)}{\partial \phi}\right|_{s}\left(1+\frac{e^{2}}{C_{0} \Delta}-\frac{f_{\mathrm{XC}}(0)}{\Delta}\right)^{-1},
$$

where $C_{0}$ is the classical capacitance defined via

$$
\frac{e^{2}}{C_{0}}=\frac{1}{V^{2}} \int d^{3} r \int d^{3} r^{\prime} \frac{e^{2}}{\left|\mathbf{r}-\mathbf{r}^{\prime}\right|},
$$

and, similarly, $f_{\mathrm{XC}}(0)$ is the volume average of $f_{\mathrm{XC}}\left(\left|\mathbf{r}-\mathbf{r}^{\prime}\right|\right)$. The derivative with the $s$ subscript is taken at constant self-consistent potential. We multiply both sides of Eq. (63) by $\delta N(\phi)$ and take the disorder average to obtain

$$
\frac{\partial \overline{[\delta N(\phi)]^{2}}}{\partial \phi}=\left.\frac{\partial \overline{[\delta N(\phi)]^{2}}}{\partial \phi}\right|_{s}\left(1+\frac{e^{2}}{C_{0} \Delta}-\frac{f_{\mathrm{XC}}(0)}{\Delta}\right)^{-1} \text {. }
$$

The derivative term on the right-hand side can be assumed to have the same value as in a noninteracting system. The factor within parentheses is the desired interaction renormalization, which arises from the variation of the self-consistent potential in response to the changing flux. If $e^{2} / C_{0} \gg \Delta, f_{\mathrm{XC}}(0)$ as is expected, since the former scales as the inverse of the length of the ring, while the latter scale as the inverse of the volume, we see that this factor is much smaller than 1, resulting in a strong suppression of fluctuations in the particle number.

Next we calculate the interaction dependence of the thermodynamic compressibility 


$$
\frac{\partial \mu}{\partial N}=\frac{1}{V^{2}} \int d^{3} r \int d^{3} r^{\prime} \chi^{-1}\left(\mathbf{r}, \mathbf{r}^{\prime}\right),
$$

where $\chi^{-1}\left(\mathbf{r}, \mathbf{r}^{\prime}\right)$. is the inverse linear density-density response function, and the integral runs over the volume of the ring. This formula is the generalization for a finite ring of the well-known compressibility sum rule ${ }^{19}$ for the electron gas. The density-density response function can be expressed, in the generalized random-phase approximation, as

$$
\chi^{-1}\left(\mathbf{r}, \mathbf{r}^{\prime}\right)=\chi_{s}^{-1}\left(\mathbf{r}, \mathbf{r}^{\prime}\right)-\frac{e^{2}}{\left|\mathbf{r}-\mathbf{r}^{\prime}\right|}-f_{\mathrm{XC}}\left(\left|\mathbf{r}-\mathbf{r}^{\prime}\right|\right)
$$

where $\chi_{s}\left(\mathbf{r}, \mathbf{r}^{\prime}\right)$ is the "Lindhard" response function of a noninteracting system with energy levels given by the Kohn-Sham equation. The physical content of this equation is that the response of the system to an external perturbing potential is equal to the response of a noninteracting system (with energy levels determined by the Kohn-Sham equation) to an effective potential, which is the sum of the external perturbing potential plus the selfconsistently induced Hartree and exchange-correlation potentials. The standard random-phase approximation is obtained by setting $f_{\mathrm{XC}}=0$ in Eq. (67).

The volume integral of $\chi_{s}^{-1}$ yields the inverse of the one-particle density of states at the Fermi surface

$$
\frac{1}{V^{2}} \int d^{3} r \int d^{3} r^{\prime} \chi_{s}^{-1}\left(\mathbf{r}, \mathbf{r}^{\prime}\right) \sim-\Delta
$$

Thus we obtain the renormalization of the compressibility,

$$
\left[\frac{\partial \mu}{\partial N}\right]=\left(\frac{\partial^{2} \Omega}{\partial \mu^{2}}\right]_{s}^{-1}\left[1+\frac{e^{2}}{C_{0} \Delta}-\frac{f_{\mathrm{XC}}(0)}{\Delta}\right) .
$$

Comparing Eqs. (69) and (65) we see that the interaction renormalizations of the compressibility and of the number variance cancel each other exactly. We thus come to the important conclusion that the part of the persistent current which is due to fluctuations of the chemical potential is given by Eq. (57), irrespective of whether the Coulomb interaction is taken into account or not. Since this correction scales as $1 / L_{1}^{2} L$ (whereas the "grand canonical" current scales as $l / L^{2}$ ) it seems unlikely that it captures the important physics of average persistent currents in ensembles of mesoscopic rings.

\section{ACKNOWLEDGMENTS}

This work has been supported by NSF Grant No. DMR-9100988. I acknowledge numerous important discussions with Peter Kopietz. I thank Professor V. Ambegaokar for kindly providing some unpublished material. Thanks are also due to Professor A. W. Overhauser and to Michael Geller for many useful comments.
${ }^{1}$ L. P. Lévy et al., Phys. Rev. Lett. 64, 2074 (1990).

${ }^{2}$ V. Chandrasekhar et al., Phys. Rev. Lett. 67, 3578 (1991).

${ }^{3}$ D. Mailly, C. Chapelier, and A. Benoit, Phys. Rev. Lett. 70, 2020 (1993).

${ }^{4}$ M. Büttiker, Y. Imry, and R. Landauer, Phys. Lett. 96A, 365 (1983).

${ }^{5}$ Ho-Fai Cheung, Y. Gefen, E. K. Riedel, and Wei-Heng Shih, Phys. Rev. B 37, 6050 (1988).

${ }^{6}$ Heléne Bouchiat and Gilles Montambaux, J. Phys (Paris) 50, 2695 (1989).

${ }^{7}$ B. L. Altshuler, Y. Gefen, and Y. Imry, Phys. Rev. Lett. 66, 88 (1991). See also B. L. Altshuler, in Nanostructures and Mesoscopic Phenomena, edited by W. P. Kirk and M. A. Reed (Academic, San Diego, 1992).

${ }^{8}$ F. von Oppen and E. K. Riedel, Phys. Rev. Lett. 66, 84 (1991).

${ }^{9}$ V. Ambegaokar and U. Eckern, Phys. Rev. Lett. 65, 381 (1990).

${ }^{10}$ A. Schmid, Phys. Rev. Lett. 66, 80 (1991).

${ }^{11}$ P. Kopietz, Phys. Rev. Lett. 70, 3123 (1993).
${ }^{12}$ For the details of the calculations, see R. A. Smith, Ph.D. thesis, Cornell University, 1993, Chaps. 7 and 8.

${ }^{13}$ V. Ambegaokar and U. Eckern, Phys. Rev. Lett. 67, 3192 (1991).

${ }^{14}$ B. L. Altshuler and A. G. Aronov, in Electron-electron Interactions in Disordered Systems, edited by A. L. Efros and M. Pollack (North-Holland, Amsterdam, 1985), Chap. 1.

${ }^{15}$ A. A. Abrikosov, L. P. Gorkov, and I. E. Dzyaloshinski, Methods of Quantum Field Theory in Statistical Physics (Dover, New York, 1963), Chap. 39, Sec. 2.

${ }^{16}$ P. Hohenberg and W. Kohn, Phys. Rev. 136, B861 (1964); W. Kohn and L. J. Sham, Phys. Rev. 140, A1133 (1965).

${ }^{17}$ N. D. Mermin, Phys. Rev. 137, A1441 (1965).

${ }^{18}$ R. M. Dreizler and E. K. U. Gross, Density Functional Theory (Springer-Verlag, Berlin, 1991), Chap. 7, Sec. 2.

${ }^{19}$ P. Nozières, Theory of Interacting Fermi Systems (Benjamin, New York, 1966). 